\title{
A 10-gene expression signature of Notch pathway predicts recurrence in ovarian carcinoma
}

\author{
FANG $\mathrm{CHEN}^{1}$ and NAIFU LIU ${ }^{2}$ \\ ${ }^{1}$ Department of Obstetrics and Gynecology, Weifang People's Hospital, Weifang, Shandong 261000; \\ ${ }^{2}$ Department of Gynecological Oncology, Shandong Cancer Hospital and Institute, Jinan, Shandong 250117, P.R. China
}

Received September 14, 2014; Accepted June 8, 2015

DOI: $10.3892 / \mathrm{ol} .2015 .3382$

\begin{abstract}
Patients with ovarian carcinoma are at high risk of tumor recurrence. In the present study, 81 Notch pathway genes were selected to find recurrence-related genes in The Cancer Genome Atlas dataset. A 10-gene signature (FZD4, HES1, PSEN2, JAG2, PPARG, FOS, HEY1, CDC16, MFNG, and EP300) was identified and validated that is associated with recurrence-free survival time, but not with overall survival time, in the TCGA dataset and in other two independent datasets, GSE9891 and GSE30161. This gene signature gave a significant performance in discriminating patients at high risk of recurrence from those at low risk, as measured by the area under the receiver operating characteristic curve. Cox proportional hazards regression analyses demonstrated that the prognostic value of this 10-gene set is independent of other clinical variables in all three datasets. The potential as a biomarker for predicting high- and low-risk subgroups for recurrence in ovarian cancer patients deserves further investigation in prospective patient cohorts in the future.
\end{abstract}

\section{Introduction}

Ovarian cancer is one of the most lethal malignant gynecological cancers worldwide (1). Compared with other cancers in women, ovarian carcinoma confers a relatively high risk of recurrence. Although there is a high initial response rate to standard surgery and chemotherapy, 30-40\% of patients relapse within one year $(2,3)$. Therefore, the prediction of patients at a high risk of recurrence may provide novel therapeutic avenues to improve their outcomes. Although common clinicopathological parameters, such as stage and histological grade, and several biomarkers were proposed

Correspondence to: Dr Naifu Liu, Department of Gynecological Oncology, Shandong Cancer Hospital and Institute, 440 Jiyan Road, Jinan, Shandong 250117, P.R. China

E-mail: naifuliu@163.com

Key words: ovarian carcinoma, recurrence, gene signature, Notch pathway, prognosis for recurrence prediction, these factors demonstrated insufficient sensitivity and specificity (4). Thus, there is an urgent requirement to identify novel markers or models to increase the power of recurrence prediction for patients with ovarian carcinoma.

The Notch pathway and its abundant associated genes comprise a complicated network, which plays a significant role in the progressive growth of tumor cells in multiple cancer types (5). The Notch pathway alterations are prevalent and significantly associated with poor outcomes, including early recurrence in ovarian carcinoma $(6,7)$. We speculate that Notch pathway associated molecular signatures may be useful for characterizing ovarian carcinomas at high risk of recurrence. In the present study, a 10-gene Notch pathway signature is defined that may assist in improved predictions of recurrence in ovarian carcinoma patients.

\section{Materials and methods}

Datasets. Three ovarian carcinoma gene expression datasets [The Cancer Genome Atlas (TCGA), GSE9891 and GSE30161] with documented recurrence information were selected for analysis in the present study (8-10). The expression data together with the curated and documented clinical metadata were extracted by the R curated Ovarian Data Bioconductor package, as previously described (11). Microarray platforms used in these datasets were Affy HT U133a (TCGA) and Affy U133 Plus 2.0 (GSE9891 and GSE30161). TCGA dataset comprises the whole-genome mRNA expression data of 522 ovarian carcinoma samples. The GSE9891 dataset comprises the gene expression microarray data of 275 ovarian carcinoma samples, including 40 early-stage and 257 late-stage tumors. The GSE30161 dataset was generated from 58 late-stage ovarian cancer samples, and 5 arrays were excluded due to the lack of complete recurrence information.

Finding of genes correlated with recurrence in TCGA ovarian carcinoma dataset. The expression data of a subset of 81 Notch pathway-associated genes (including core Notch pathway members, Notch pathway target genes, genes that crosstalk with Notch pathway and other genes involved in Notch pathway) were selected from TCGA ovarian carcinoma dataset. Cox proportional hazards model was used to test whether the gene expression of a particular gene significantly influenced 
Table I. Notch pathway genes correlated with recurrence in The Cancer Genome Atlas ovarian carcinoma dataset.

\begin{tabular}{|c|c|c|c|c|c|}
\hline $\begin{array}{l}\text { Notch pathway } \\
\text { genes }\end{array}$ & Gene names & Accession no. & Hazard ratio & FDR & $\begin{array}{l}\text { Permutation } \\
\text { P-value }\end{array}$ \\
\hline FZD4 & Frizzled family receptor 4 & NM_012193 & 0.786 & 0.0466 & 0.0010 \\
\hline HES 1 & $\begin{array}{c}\text { Hairy and enhancer of split } 1, \\
\text { (Drosophila) }\end{array}$ & NM_005524 & 0.874 & 0.259 & 0.0091 \\
\hline PSEN2 & Presenilin 2 (Alzheimer disease 4) & NM_000447 & 0.471 & 0.259 & 0.0094 \\
\hline JAG2 & Jagged 2 & NM_002226 & 0.819 & 0.286 & 0.0241 \\
\hline PPARG & $\begin{array}{l}\text { Peroxisome proliferator-activated } \\
\text { receptor gamma }\end{array}$ & NM_005037 & 0.741 & 0.286 & 0.0284 \\
\hline FOS & $\begin{array}{l}\text { FBJ murine osteosarcoma viral } \\
\text { oncogene homolog }\end{array}$ & NM_005252 & 1.094 & 0.286 & 0.0266 \\
\hline HEY1 & $\begin{array}{l}\text { Hairy/enhancer-of-split related } \\
\text { with YRPW motif } 1\end{array}$ & NM_001040708 & 0.879 & 0.286 & 0.0298 \\
\hline CDC16 & Cell division cycle 16 & NM_001078645 & 1.202 & 0.286 & 0.0296 \\
\hline MFNG & $\begin{array}{l}\text { MFNG O-fucosylpeptide 3- } \beta \text { - } \\
\text { N-acetylglucosaminyltransferase }\end{array}$ & NM_001166343 & 1.323 & 0.286 & 0.0357 \\
\hline EP300 & E1A-binding protein $\mathrm{p} 300$ & NM_001429 & 0.818 & 0.321 & 0.0429 \\
\hline
\end{tabular}

FDR, false discovery rate.

recurrence using the BRB-Arraytools software (12). The tests were performed at a significance threshold of univariate tests of 0.05 using permutation tests. The number of permutation tests was set as 10,000 .

Recurrence-free survival (RFS) and overall survival (OS) time prediction based on the supervised principal components method using the 10-Notch pathway gene signature. After subseting the gene expression data using the 10-Notch pathway gene signature, recurrence and survival risk prediction in TCGA, GSE9891 and GSE30161 datasets were performed based on principal components using the BRB-ArrayTools software. 10-fold cross validation was selected, and the number of principal components was set as 2 . The prognostic index percentile was used to separate arrays into high- and low-risk groups.

Statistical analysis. Distributions of RFS and OS were assessed using the Kaplan-Meier curve method and evaluated by the log-rank test. Multivariate analyses of prognostic factors were based on the Cox proportional hazards model. The receiver operating characteristic (ROC) curve was constructed using $\mathrm{R}$ package survival ROC and determined by permutation testing. The difference in clinicopathological characteristics between the high- and low-risk subgroups was determined by $\chi^{2}$ test. All the statistical analyses were performed with Medcalc 11.4 Software unless otherwise specified. $\mathrm{P}<0.05$ was considered to indicate a statistically significant difference.

\section{Results}

We hypothesized that Notch pathway genes correlated with recurrence may be clinically useful to differentiate between high- and low-risk ovarian carcinoma tumors. To investigate this, the BRB-Arraytool package was used to find Notch pathway genes with expression that was correlated with RFS time by univariate tests. As shown in Table I, using a significance threshold at 0.05 , a list of 10 Notch pathway genes was determined to be associated with RFS time in TCGA ovarian carcinoma dataset.

Using a principal components model based on these 10 Notch pathway genes as a signature, a predictor was generated and TCGA ovarian cancer samples were classified into high-risk $(n=263)$ and low-risk $(n=259)$ subgroups. As shown in Fig. 1A, in TCGA dataset, the high-risk ovarian carcinomas demonstrated a significantly shorter RFS time than the low-risk cases (hazard ratio, 1.3656; 95\% confidence interval, 1.0739-1.7364; $\mathrm{P}=0.0104)$. In order to evaluate the performance of this novel gene signature, its performance in predicting recurrence in two other independent ovarian carcinoma datasets, GSE9891 and GSE30161, was further validated. As shown in Fig. 1B and C, the signature was independently predictive of recurrence in the two validation datasets. In addition, this gene signature gave a significant value for the area under the curve when discriminating between high- and low-risk ovarian carcinomas in all three datasets (TCGA, $\mathrm{P}=0.0167$; GSE9891, $\mathrm{P}=0.04$; GSE30161, $\mathrm{P}=0.01$ ) (Fig. 1D-F).

Next, the association between the predicted recurrence risk subgroups and the known prognostic factors was analyzed. In GSE9891, but not the other two datasets, the high recurrence risk subgroup exhibited a significant association with stage and debulking level (Table II). The multivariate Cox proportional hazards regression analyses found that the prognostic value on recurrence of the 10-Notch gene signature was independent of other known predictors in all three datasets (Table III).

Finally, it was evaluated whether this 10 -gene signature could predict OS time for ovarian carcinomas. In contrast to RFS, no significant difference was found in OS time between the high- and low- risk subgroups in all three datasets (Fig. 2). 
A

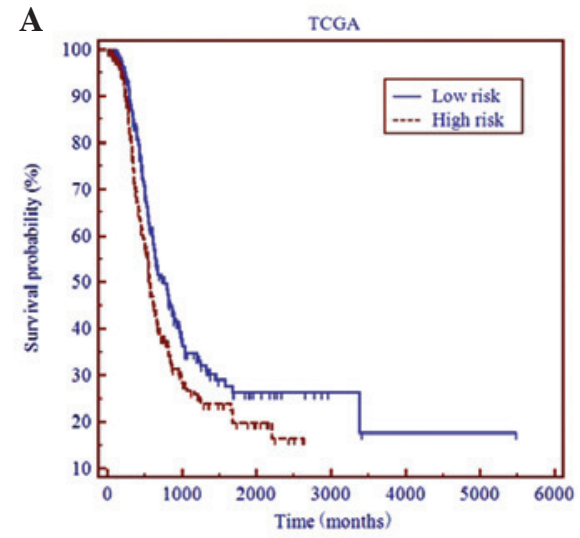

D

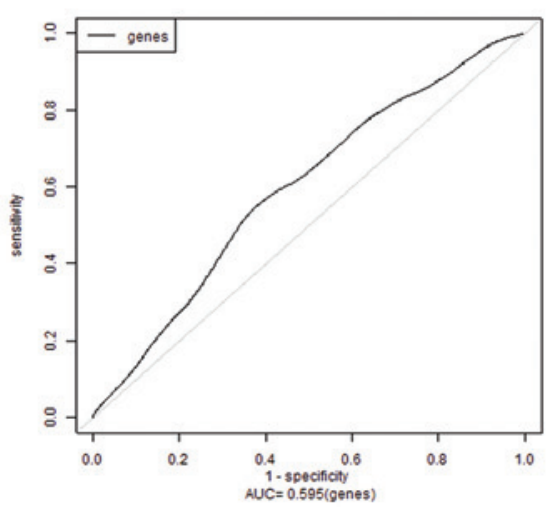

B

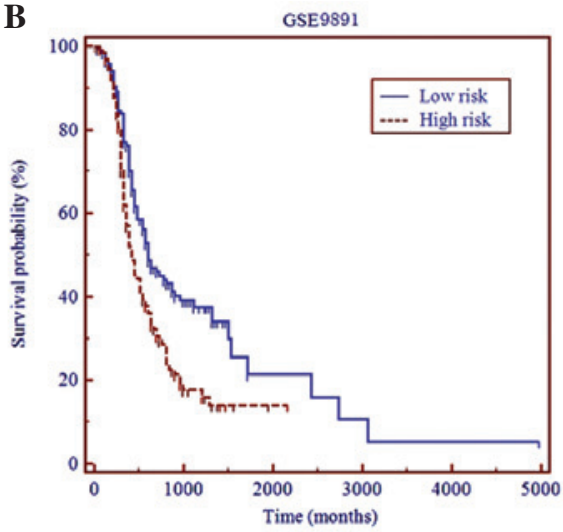

E

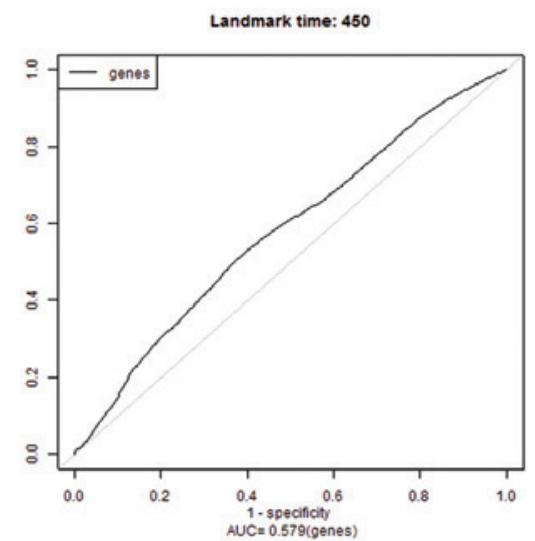

C

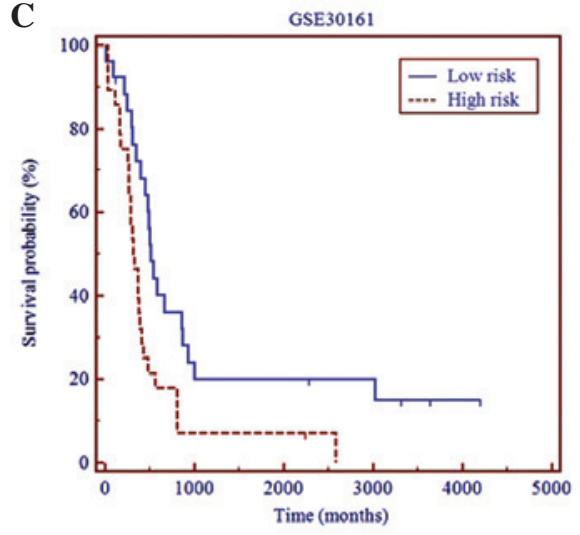

F

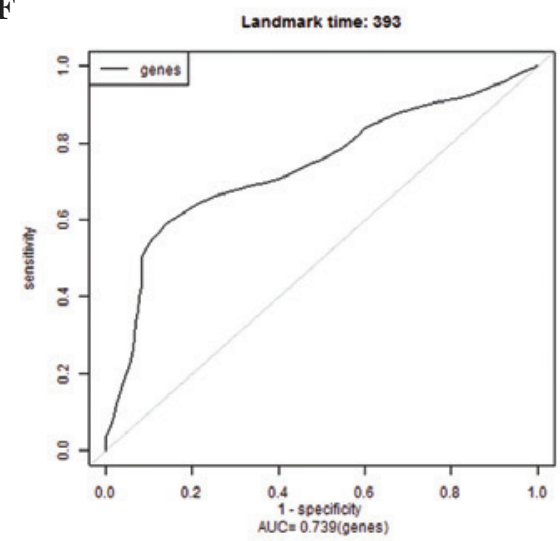

Figure 1. Recurrence prediction and receiver operating characteristic curves in 3 ovarian carcinoma gene expression datasets based on a 10-Notch pathway gene classifier. High-risk subgroups showed a significantly shorter recurrence-free survival time than low-risk groups in (A) The Cancer Genome Atlas (TCGA), (B) GSE9891 and (C) GSE30161 datasets. This gene signature gave an area under the curve (AUC) of (D) 0.595 in TCGA dataset; (E) 0.579 in the GSE9891 dataset and (F) 0.739 in the GSE30161 dataset.

A

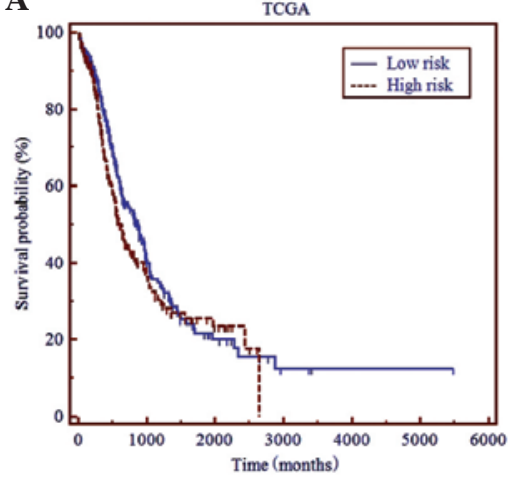

B

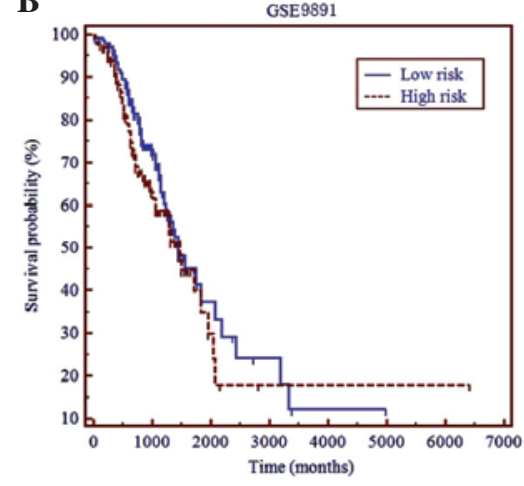

C

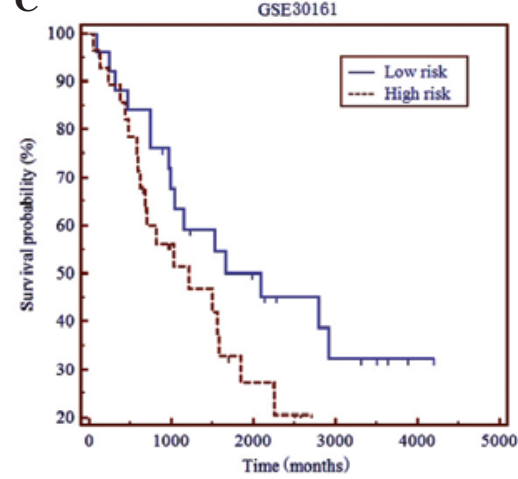

Figure 2. Overall survival (OS) time prediction in 3 ovarian carcinoma gene expression datasets based on the 10-Notch pathway gene classification. No difference was found in OS time between high- and low-risk subgroups in (A) The Cancer Genome Atlas (TCGA) dataset; (B) the GSE9891 dataset and (C) the GSE30161 dataset.

\section{Discussion}

In the current study, 10 Notch pathway genes were identified to significantly correlate with recurrence in ovarian carcinoma. This 10-gene signature could classify ovarian carcinoma into high- and low-risk recurrence subgroups, and showed a significant performance to predict recurrence in independent cohorts. Moreover, the prognostic value of this gene signature is independent of the common clinicopathological predictors of ovarian cancers.
Prognostic gene signatures based on microarray data have been recently developed to identify subgroups with a more aggressive phenotype or poor outcomes in ovarian carcinoma. For example, Gillet et al (13) found 3 multidrug resistance gene signatures with a statistically significant correlation with OS and progression-free survival time. Cheon et al (14) identified and validated a 10 -gene signature regulated by transforming growth factor- $\beta$ signaling that is associated with poor OS time in patients with high-grade serous ovarian cancer. Compared with the previous gene signatures, the present 10 -gene classifier 
Table II. Difference in clinicopathological characteristics between high- and low-risk recurrence subgroups defined by a 10-Notch pathway gene signature in 3 ovarian carcinoma gene expression datasets. ${ }^{\mathrm{a}}$

\begin{tabular}{|c|c|c|c|}
\hline Characteristics & Low risk & High risk & P-value \\
\hline TCGA dataset & & & 0.9589 \\
\hline Number & 259 & 263 & \\
\hline \multicolumn{4}{|l|}{ Grade } \\
\hline $1-2$ & 33 & 34 & \\
\hline 3 & 224 & 220 & \\
\hline Stage & & & 0.6486 \\
\hline Early & 18 & 22 & \\
\hline Late & 240 & 239 & \\
\hline Debulking & & & 0.1067 \\
\hline Optimal & 184 & 167 & \\
\hline Suboptimal & 52 & 68 & \\
\hline \multicolumn{4}{|c|}{ GSE9891 dataset } \\
\hline Number & 142 & 133 & 0.0114 \\
\hline \multicolumn{4}{|l|}{ Grade } \\
\hline $1-2$ & 68 & 44 & \\
\hline 3 & 71 & 89 & \\
\hline Stage & & & 0.0008 \\
\hline Early & 31 & 9 & \\
\hline Late & 111 & 123 & \\
\hline Debulking & & & 0.8884 \\
\hline Optimal & 41 & 41 & \\
\hline Suboptimal & 83 & 77 & \\
\hline \multicolumn{4}{|c|}{ GSE30161 dataset } \\
\hline Number & 26 & 28 & 1.0000 \\
\hline \multicolumn{4}{|l|}{ Grade } \\
\hline $1-2$ & 11 & 10 & \\
\hline 3 & 14 & 15 & \\
\hline Debulking & & & 0.9485 \\
\hline Optimal & 14 & 15 & \\
\hline Suboptimal & 10 & 13 & \\
\hline
\end{tabular}

${ }^{\mathrm{a} C}$ Cases with unavailable clinicopathological data were not included.

exhibited a better performance on predicting recurrence, but not OS time for ovarian carcinoma. Moreover, for the first time, a Notch pathway gene signature is reported to have clinical significance in the prognosis prediction for patients with ovarian carcinoma. The use of this 10-Notch pathway gene signature should be investigated in prospective patient cohorts in the future.

Of the 10 Notch pathway genes identified in the present study, the functional and clinical significance has only been investigated in FZD4, HES1, PPARG and FOS. Only FZD4 has been found to be associated with recurrence in a previous study. Dai et al (15) found that DNA methylation and reduced expression of FZD4 are indicators of early disease relapse in
Table III. Multivariate Cox proportional hazards regression analyses on recurrence-free survival time in 3 ovarian carcinoma gene expression datasets.

\begin{tabular}{lccc}
\hline Covariate & P-value & $\operatorname{Exp}(\mathrm{b})$ & 95\% CI of Exp(b) \\
\hline TCGA dataset & & & \\
Grade & 0.5624 & 1.1112 & $0.7792-1.5848$ \\
Stage & 0.0155 & 2.0291 & $1.1473-3.5887$ \\
Debulking & 0.6801 & 0.9404 & $0.7032-1.2575$ \\
Predicted risk & 0.0083 & 1.4119 & $1.0944-1.8215$ \\
GSE9891 dataset & & & \\
Grade & 0.6509 & 0.9377 & $0.7106-1.2373$ \\
Stage & 0.0001 & 5.0027 & $2.3040-10.8624$ \\
Debulking & 0.0010 & 0.5829 & $0.4228-0.8035$ \\
Predicted risk & 0.0382 & 1.3984 & $1.0200-1.9171$ \\
GSE30161 dataset & & & \\
Grade & 0.7870 & 1.0997 & $0.5537-2.1841$ \\
Debulking & 0.0160 & 0.4235 & $0.2113-0.8488$ \\
Predicted risk & 0.0088 & 2.3312 & $1.2413-4.3781$ \\
\hline
\end{tabular}

TCGA, The Cancer Genome Atlas; CI, confidence interval.

ovarian tumors, which is consistent with the present finding that FZD4 gene expression is negatively associated with recurrence in ovarian carcinoma. Increased expression of HES1 and PPARG were validated to be predictors for poor OS in ovarian cancers $(16,17)$. High expression of FOS was significantly associated with advanced clinical stage and chemoresistance (18). Considering that this 10-gene signature has clinical significance in classifying different subgroups with high and low recurrence risk, it is also possible that these Notch pathway genes may act as important mediators during ovarian carcinogenesis, and may represent novel therapeutic targets. Therefore, the biological significance of these 10 Notch signaling genes also deserves further investigation.

In conclusion, in the present study, a novel Notch pathway gene signature that is useful for predicting recurrence in ovarian cancers was developed. If prospectively validated, it would provide a reference for informing treatment decisions for patients with ovarian carcinoma. The biological significance of this signature and its potential as a biomarker also deserve further investigation in future studies.

\section{References}

1. Lowe KA, Chia VM, Taylor A, O'Malley C, Kelsh M, Mohamed M, Mowat FS and Goff B: An international assessment of ovarian cancer incidence and mortality. Gynecol Oncol 130: 107-114, 2013.

2. Foley OW, Rauh-Hain JA and del Carmen MG: Recurrent epithelial ovarian cancer: an update on treatment. Oncology (Williston Park) 27: 288-294, 298, 2013.

3. Geurts SM, de Vegt F, van Altena AM, van Dijck JA, Tjan-Heijnen VC, Verbeek AL and Massuger LF: Considering early detection of relapsed ovarian cancer: a review of the literature. Int J Gynecol Cancer 21: 837-845, 2011.

4. Santillan A, Garg R, Zahurak ML, Gardner GJ, Giuntoli RL II, Armstrong DK and Bristow RE: Risk of epithelial ovarian cancer recurrence in patients with rising serum CA-125 levels within the normal range. J Clin Oncol 23: 9338-9343, 2005. 
5. Takebe N, Nguyen D and Yang SX: Targeting notch signaling pathway in cancer: Clinical development advances and challenges. Pharmacol Ther 141: 140-149, 2014.

6. Rose SL, Kunnimalaiyaan M, Drenzek J and Seiler N: Notch 1 signaling is active in ovarian cancer. Gynecol Oncol 117: 130-133, 2010.

7. Jung SG, Kwon YD, Song JA, Back MJ, Lee SY, Lee C, Hwang YY and An HJ: Prognostic significance of Notch 3 gene expression in ovarian serous carcinoma. Cancer Sci 101: 1977-1983, 2010.

8. Cancer Genome Atlas Research Network: Integrated genomic analyses of ovarian carcinoma. Nature 474: 609-615, 2011.

9. Tothill RW, Tinker AV, George J, Brown R, Fox SB, Lade S, Johnson DS, Trivett MK, Etemadmoghadam D, Locandro B, et al: Novel molecular subtypes of serous and endometrioid ovarian cancer linked to clinical outcome. Clin Cancer Res 14: 5198-5208, 2008.

10. Ferriss JS, Kim Y, Duska L, Birrer M, Levine DA, Moskaluk C, Theodorescu D and Lee JK: Multi-gene expression predictors of single drug responses to adjuvant chemotherapy in ovarian carcinoma: Predicting platinum resistance. PLoS One 7: e30550, 2012.

11. Ganzfried BF, Riester M, Haibe-Kains B, Risch T, Tyekucheva S, Jazic I, Wang XV, Ahmadifar M, Birrer MJ, Parmigiani G, et al: CuratedOvarianData: Clinically annotated data for the ovarian cancer transcriptome. Database (Oxford) 2013: bat013, 2013.

12. Simon R, Lam A, Li MC, Ngan M, Menenzes S and Zhao Y: Analysis of gene expression data using BRB-ArrayTools. Cancer Inform 3: 11-17, 2007.
13. Gillet JP, Wang J, Calcagno AM, Green LJ, Varma S, Bunkholt Elstrand M, Trope CG, Ambudkar SV, Davidson B, et al: Clinical relevance of multidrug resistance gene expression in ovarian serous carcinoma effusions. Mol Pharm 8: 2080-2088, 2011.

14. Cheon DJ, Tong Y, Sim MS, Dering J, Berel D, Cui X, Lester J, Beach JA, Tighiouart M, Walts AE, et al: A collagen-remodeling gene signature regulated by TGF- $\beta$ signaling is associated with metastasis and poor survival in serous ovarian cancer. Clin Cancer Res 20: 711-723, 2014.

15. Dai W, Teodoridis JM, Zeller C, Graham J, Hersey J, Flanagan JM, Stronach E, Millan DW, Siddiqui N, Paul J, et al: Systematic CpG islands methylation profiling of genes in the wnt pathway in epithelial ovarian cancer identifies biomarkers of progression-free survival. Clin Cancer Res 17: 4052-4062, 2011.

16. Wang X, Fu Y, Chen X, Ye J, Lü B, Ye F, Lü W and Xie X: The expressions of bHLH gene HES1 and HES5 in advanced ovarian serous adenocarcinomas and their prognostic significance: A retrospective clinical study. J Cancer Res Clin Oncol 136: 989-996, 2010.

17. Ivan $\mathrm{C}, \mathrm{Hu} \mathrm{W}$, Bottsford-Miller J, Zand B, Dalton HJ, Liu T, Huang J, Nick AM, Lopez-Berestein G, Coleman RL, et al: Epigenetic analysis of the Notch superfamily in high-grade serous ovarian cancer. Gynecol Oncol 128: 506-511, 2013.

18. Kang KW, Lee MJ, Song JA, Jeong JY, Kim YK, Lee C, Kim TH, Kwak KB, Kim OJ and An HJ: Overexpression of goosecoid homeobox is associated with chemoresistance and poor prognosis in ovarian carcinoma. Oncol Rep 32: 189-198, 2014. 